\title{
ON COSET REPRESENTATIVES IN GROUPS
}

\section{OYSTEIN ORE}

1. In the following we denote by $G$ some fixed group with the subgroups $H$ and $K$. In a right coset expansion of $G$ with respect to $H$ one may possibly select the representatives for the cosets such that they may also be used for representatives in a left coset expansion with respect to $K$

$$
G=\sum g H=\sum K g .
$$

We then say that there exist common representatives for the two expansions. The case $H=K$ is of special interest. Then

$$
G=\sum g H=\sum H g
$$

and $G$ has common left and right representatives with respect to $H$. It has been shown by Miller [1] and Chapman [2] that expansions (1.2) exist when $H$ is finite. According to Scorza [3] expansions (1.1) exist when $H$ and $K$ are finite with the same order. In the present paper we shall point out some general cases in which such expansions must exist for infinite subgroups $H$ and $K$.

2. Let

$$
G=\sum K d H
$$

be the double coset expansion of $G$ with respect to $H$ and $K$. It is readily seen that an expansion (1.1) can only exist when there is such an expansion for each of the double cosets in (2.1). A coset expansion of $K d H$ will have the form

$$
K d H=\sum K d h=\sum k d H, h \in H, k \in K .
$$

Here any two cosets $K d h$ and $k d H$ have a nonvoid intersection since the element $k d h$ belongs to both. When there exist common representatives for the two expansions (2.2) of $K d H$ the cardinal number of cosets of $H$ and $K$ is the same. Conversely, if this is the case there is a one-to-one correspondence

$$
H_{i} \rightleftarrows K_{i}
$$

between the two families of cosets. For each $i$ one can select an arbitrary 1958.

Received by the editors November 29, 1957 and, in revised form, February 24,

1 This research was supported by a grant from the National Science Foundation. 


$$
g_{i} \in H_{i} \cap K_{i}
$$

and these elements then form a system of common representatives in (2.2).

In (2.2) two elements $h$ and $h_{1}$ define the same $K$-coset

$$
K d h=K d h_{1}
$$

if and only if

$$
h_{1} h^{-1} \in H \cap d^{-1} K d .
$$

Thus the distinct $K$-cosets in $K d H$ correspond in a one-to-one manner to the cosets in the expansion

$$
H=\sum D_{H} \cdot h_{i}, \quad D_{H}=H \cap d^{-1} K d .
$$

Analogously the distinct $H$-cosets in $K d H$ correspond in a one-to-one manner to the cosets in the expansion

$$
K=\sum k_{i} D_{K}, \quad D_{K}=K \cap d H d^{-1} .
$$

This leads immediately to:

THEOREM 2.1. A necessary and sufficient condition that the group $G$ have common representatives for expansion with respect to $H$ and $K$ is that for all $d \in G$ the two indices

$$
\left[H / D_{H}\right]=\left[K / D_{K}\right]
$$

have the same cardinal number. Here the groups $D_{H}$ and $D_{K}$ are defined in (2.3) and (2.4).

For $H=K$ the condition for common left and right representatives for a group $H$ becomes

$$
\left[H / H \cap d^{-1} H d\right]=\left[H / H \cap d H d^{-1}\right] .
$$

3. The groups $D_{H}$ and $D_{K}$ are conjugate

$$
d D_{H} d^{-1}=D_{K} .
$$

Thus we conclude:

THEOREM 3.1. The group $G$ has common representatives for the subgroups $H$ and $K$ when these groups have the property: If $H_{1}$ and $K_{1}$ are conjugate subgroups in $G$ such that

$$
H \supset H_{1}, \quad K \supset K_{1}
$$

then

$$
\left[H / H_{1}\right]=\left[K / K_{1}\right]
$$


The following criterion depends only on $H$ and $K$ and is independent of $G$ :

THEOREM 3.2. There exist common representatives for $H$ and $K$ if (3.2) holds for every pair of isomorphic subgroups $H_{1}$ and $K_{1}$ of $H$ and $K$ respectively.

When applied to $H=K$ we obtain:

THEOREM 3.3. $G$ has common left and right coset representatives for $H$ if any two conjugate subgroups of $G$ contained in $H$ have the same index in $H$; thus in particular if $H$ has the property that any two isomorphic subgroups in it always have the same index.

These results evidently include the previously mentioned results by Miller, Chapman and Scorza for the case where $H$ and $K$ are finite.

4. Let $d \in G$. A subgroup $A$ is $d$-invariant if

$$
d A d^{-1}=A \text {. }
$$

Every subgroup $H$ of $G$ contains a maximal $d$-invariant subgroup $I_{d}(H)$ given by the formula

$$
I_{d}(H)=\wedge H_{i}, \quad i=0, \pm 1, \pm 2, \cdots
$$

as the intersection of the conjugates

$$
H_{i}=d^{i} H d^{-i}
$$

The formula (4.1) shows that

$$
I_{d}(H)=I_{d^{-1}}(H) .
$$

All $H_{i}$ in (4.2) have the same maximal $d$-invariant subgroup $I_{d}(H)$. For two arbitrary subgroups $A$ and $B$

$$
I_{d}(A \cap B)=I_{d}(A) \cap I_{d}(B) .
$$

From the index relation

$$
\begin{aligned}
& {\left[A \cap B / I_{d}(A \cap B)\right]} \\
& \quad=\left[A \cap B / I_{d}(A) \cap B\right] \cdot\left[I_{d}(A) \cap B / I_{d}(A) \cap I_{d}(B)\right]
\end{aligned}
$$

one concludes that

$$
\left[A \cap B / I_{d}(A \cap B)\right] \leqq\left[A / I_{d}(A)\right] \cdot\left[B / I_{d}(B)\right] .
$$

Let us return to the condition (2.5) for the existence of common representatives. According to (3.1) it may be written

$$
\left[H / H \cap d^{-1} K d\right]=\left[d^{-1} K d / H \cap d^{-1} K d\right] .
$$


We denote the maximal $d$-invariant subgroups of $H$ and $K$ respectively by

$$
I_{d}(H), \quad I_{d}(K) .
$$

From (4.4) and the preceding remarks we obtain

$I_{d}(H \cap K)=I_{d}(H) \cap I_{d}(K)=I_{d}(H) \cap I_{d}\left(d^{-1} K d\right)=I_{d}\left(H \cap d^{-1} K d\right)$.

Thus a consequence of (4.6) is

$$
\left[H / I_{d}(H \cap K)\right]=\left[d^{-1} K d / I_{d}(H \cap K)\right]
$$

and so we have:

ThEOREM 4.1. A necessary condition for the existence of common representatives for $H$ and $K$ is that for any $d \in G$

$$
\left[H / I_{d}(H \cap K)\right]=\left[K / I_{d}(H \cap K)\right] .
$$

Assume that the indices

$$
\left[H / I_{d}(H)\right], \quad\left[K / I_{d}(K)\right]
$$

are finite. It follows from (4.5) that also the indices

$$
\left[H \cap K / I_{d}(H \cap K)\right], \quad\left[H \cap d^{-1} K d / I_{d}(H \cap K)\right]
$$

are finite. Thus one can conclude reversely from (4.9) to (2.5) through (4.8) and (4.6). Therefore in this case the condition (4.9) is both necessary and sufficient. But from the index relation

$$
\left[H / I_{d}(H \cap K)\right]=[H / H \cap K] \cdot\left[H \cap K / I_{d}(H \cap K)\right]
$$

and analogously for $K$ we conclude further:

THEOREM 4.2. Let $H$ and $K$ be subgroups such that the indices (4.10) are finite for every $d \in G$. Then there exist common representatives for $H$ and $K$ if and only if

$$
[H / H \cap K]=[K / H \cap K] .
$$

It is well known that when $H$ and $K$ are subgroups of $G$ of finite index then each of them contains a normal subgroup of $G$ also of finite index. We conclude that in this case the indices (4.10) are finite. But then the condition (4.11) can be replaced by

$$
[G / H]=[G / K]
$$

and so we may state:

TheOREM 4.3. When $H$ and $K$ are subgroups of finite index in $G$ then they have common representatives if and only if they have the same index in $G$. 
For $H=K$ the condition for common right and left representatives in Theorem 4.2 becomes simply that the index

$$
\left[H / I_{d}(H)\right]
$$

shall be finite. This is always fulfilled when $H$ has finite index in $G$.

5. We shall investigate the case where the index (4.12) is infinite. For $d \in G$ let $H$ and $B$ be subgroups such that

$$
H \supset B \cup d B d^{-1} \text {. }
$$

Then

$$
[H / B] \geqq\left[d B d^{-1} / B \cap d B d^{-1}\right]
$$

and consequently

$$
[H / B] \geqq\left[B / B \cap d^{-1} B d\right]
$$

We shall put

$$
I_{k}(B, d)=B \cap d^{-1} B d \cap \cdots \cap d^{-k} B d^{k} .
$$

Since

$$
d I_{k} d^{-1}=d B d^{-1} \cap I_{k-1} \subset I_{k-1}
$$

the inequality (5.2) may be applied repeatedly so that one obtains

$$
[H / B] \geqq\left[I_{k-1} / I_{k}\right] .
$$

From the index relation

$$
\left[H / I_{k}\right]=\left[H / I_{0}\right] \cdot\left[I_{0} / I_{1}\right] \cdots\left[I_{k-1} / I_{k}\right]
$$

we conclude that

$$
\left[H / I_{k}\right] \leqq[H / B]^{k+1} .
$$

Let us say that $I_{d}(H)$ in (4.1) has the finite intersection property if it is the intersection of a finite number of the conjugates (4.2). This is the case, in particular, when there is only a finite number of such conjugates. In (5.3) we next make use of the fact that for any infinite cardinal one has

$$
\alpha^{n}=\alpha, \quad n \text { finite }
$$

It then follows:

THEOREM 5.1. Let $H$ and $B$ be subgroups satisfying (5.1) and suppose that $I_{d}(B)$ has the finite intersection property. Then the indices

$$
[H / B], \quad\left[H / I_{d}(B)\right]
$$

are either both finite or both infinite and in the latter case they are equal. 
In connection with this theorem let us mention the following analogue for normal subgroups: Let $H$ be a subgroup of $G$ and $N$ the greatest normal subroup of $G$ contained in $H$; thus $N$ is the intersection of all conjugates of $H$. If $N$ can be represented as the intersection of a finite number of such conjugates the indices

$$
[G / H], \quad[G / N]
$$

are either both finite or both infinite and in the latter case

$$
[G / H]=[G / N] \text {. }
$$

We shall apply Theorem 5.1 to

$$
B=H \cap d^{-1} H d
$$

so that

$$
I_{d}(B)=I_{d}(H)
$$

It follows:

THEOREM 5.2. Let $I_{d}(H)$ have the finite intersection property. Then

$$
\left[H / I_{d}(H)\right]=\left[H / H \cap d^{-1} H d\right]
$$

when the first of these indices is infinite.

From (5.4) we obtain (2.6) by means of the observation (4.3) and so we may state:

THEOREM 5.3. The group $G$ has common left and right coset representatives for the group $H$ when for every $d \in G$ the maximal d-invariant subgroup $I_{d}(H)$ of $H$ has the finite intersection property.

The condition is satisfied when $H$ has a finite number of conjugates by transformation with the powers of any element $d \in G$. Theorem 5.3 then gives a result due to Shü [4].

\section{BiBLIOGRAPHY}

1. G. A. Miller, On a method due to Galois, Quart. J. Math. Oxford Ser. vol. 41 (1910) pp. 382-384.

2. H. W. Chapman, A note on the elementary theory of groups of finite order, Messenger of Math. vol. 42 (1913) pp. 132-134.

3. G. Scorza, A proposito di un teorema del Chapman, Bolletino della Unione Matematica Italiana vol. 6 (1927) pp. 1-6.

4. S. Shü, On the common representative system of residue classes of infinite groups, J. London Math. Soc. vol. 16 (1941) pp. 101-104.

YALE UNIVERSITY 